Exp. Anim. 57(1), 19-25, 2008

\title{
Effect of Maternal Restraint Stress on Fetal Development of ICR Mice
}

\author{
Yea Eun LEE ${ }^{1)}$, Sang Kuk BYUN ${ }^{1)}$, Sunhee SHIN'1), Ja Young JANG'1), Byong-il CHOI'), \\ Dongsun PARK ${ }^{1)}$, Jeong Hee JEON ${ }^{1)}$, Sang-Seop NAHM ${ }^{2)}$, Jong-Koo KANG ${ }^{1}$, \\ Seock-Yeon $\mathrm{HWANG}^{3)}$, Jong-Choon $\mathrm{KIM}^{4}$ ), and Yun-Bae KIM${ }^{1)}$
}

\begin{abstract}
${ }^{1)}$ College of Veterinary Medicine and Research Institute of Veterinary Medicine, Chungbuk National University, 12 Gaeshin-dong, Cheongju, Chungbuk 361-763, ${ }^{2}$ College of Veterinary Medicine, Konkuk University, 1 Hwayang-dong, Kwangjin-gu, Seoul 143-701, 3)Department of Clinical Laboratory Science, Juseong College, San 4 Deokam-ri, Naesu-eup, Cheongwon, Chungbuk 363-794, and ${ }^{4)}$ College of Veterinary Medicine, Chonnam National University, 300 Yongbong-dong, Buk-gu, Gwangju 500-757, Korea
\end{abstract}

\begin{abstract}
The present study was conducted to elucidate the susceptibility of embryos and fetuses at different gestational stages to the maternal stress in mice. Groups of pregnant ICR mice were subjected to daily 12-h restraint stress, taped in the supine position on a plastic board, on gestational days (GD) 1-4, 5-8, 9-12 and 13-16, respectively. Caesarean sections were performed on gestational day 18, and the fetuses were weighed and examined for morphological defects. During the daily restraint for 4 days, the maternal body weights markedly decreased. Although the body weights recovered gradually after termination of the stress, the recovery was not full until the final stage of pregnancy. Interestingly, restraint stress caused growth retardation of the fetuses, leading to a significant decrease in their body weights, and increased early and late resorptions of embryos and fetuses according to the stress periods. Although the preceding (GD1-4) and concurrent (GD5-8) stresses did not affect embryonic implantation, restraint stress on GD9-12 caused cleft palate. Whereas vertebral abnormalities, mainly bipartite ossification, were observed only in animals stressed on GD5-8, abnormalities of sternebrae, exhibiting asymmetric or bipartite ossification, were enhanced by the stress at all of the gestational stages. On the other hand, the incidence of other malformations including renal malposition and costal abnormalities was not increased by stress at any of the 4 stages. Taken together, the results suggest that intensive restraint stress influences the maternal body weight resulting in growth retardation and increased mortality of embryos and fetuses, in addition to gestational stage-specific ventricular dilatation, cleft palate and sternal abnormalities.
\end{abstract}

Key words: fetal development, malformations, maternal toxicity, restraint, stress

(Received 26 June 2007 / Accepted 1 August 2007)

Address corresponding: Y.B. Kim, College of Veterinary Medicine, Chungbuk National University, 12 Gaeshin-dong, Cheongju, Chungbuk 361763, Korea 


\section{Introduction}

It has been reported that maternal stress causes growth retardation, cleft palate or resorption of fetuses in rodents $[3,20,32]$. Increased production of glucocorticoids during maternal stress mediating embryo-fetal toxicities including cleft palate and skeletal malformations has been proposed as a possible mechanism [4-6, 21], and has been demonstrated that excess exposure to glucocorticoids retards fetal growth in animals and humans $[22,25,30,34]$. Subsequent studies proposed placental $11 \beta$-hydroxysteroid dehydrogenase, which converts active cortisol to inactive cortisone, as the mechanism guarding fetuses from growth-retarding effects of maternal glucocorticoids [7, 35].

There are, however, species and strain differences in the adrenocortical response to stress and in susceptibilities to glucocorticoid-induced embryo-fetal toxicities $[15,17,18,27]$. In general, mice have been found to be more susceptible to stress- or glucocorticoid-induced teratogenesis than rats $[9,15]$. Furthermore, the presence of glucocorticoid receptors on the embryonic palate was correlated across mouse strains with differential susceptibility to cleft palate induction [40].

In addition to the gestational period, the duration and intensity of stress might be rate-limiting factors in the induction of reproductive abnormalities, since implantation and organogenesis generally occur in rodents on gestational days (GD) 5-6 and 6-15, respectively. Many investigators have reported controversial results based on their stress conditions [15], such as gestational period (GD0-20), duration of stress (1.8 min-48 h) and stressors (restraints, electric shock or feed/water deprivation). These results led us to investigate the specific gestational stage susceptibilities of fetuses to maternal stress in mice.

\section{Materials and Methods}

\section{Animals}

Seven-week-old male and female ICR mice (28-30 g) were purchased from Daehan Laboratory Animal Center, Korea, and housed at the Laboratory Animal Research Center of Chungbuk National University. The animals ( $n=15$ per group) were maintained at a constant tempera- ture of $22 \pm 1{ }^{\circ} \mathrm{C}$, relative humidity of $55 \pm 10 \%$ and 12 -h light/dark cycle, and fed with standard rodent chow and purified water.

All experimental procedures were carried out in accordance with the National Institutes of Health Guide for the Care and Use of Laboratory Animals (NIH Publication No.85-23, revised in 1996), and the protocol was approved by Institutional Animal Care and Use Committee of Laboratory Animal Research Center, Chungbuk National University, Korea.

\section{Restraint stress}

Female mice were mated with males $(1: 1)$ at 18:00, and examined for vaginal plug on the following day at 08:00. Mating, if unsuccessful, was repeated for a maximum of 4 days. Successful mating was confirmed by the presence of vaginal plug, and the following $24 \mathrm{~h}$ was defined as GD0.

Pregnant mice were divided into 4 groups according to the scheduled stress periods during gestation: GD1-4 (pre-implantation), GD5-8 (peri-implantation), GD9-12 (early organogenesis) and GD13-16 (late organogenesis and development). The dams were subjected to restraint stress by 12-h taping of 4 limbs to a plastic board in a supine position $[8,21,28,29]$ under the 300 lux room light (08:00-20:00) for 4 days. The 12-h taping restraint stress was adopted for the present experiments because restraint stress longer than $12 \mathrm{~h}$ is generally required for the induction of malformation or resorption of fetuses [15]. During restraint, animals had no access to food and water. They were returned to their home cages after stress performance. Body weights were recorded prior to each stress trial. Since feed and water were inaccessible during restraint, all the mice, including animals in the control group, were kept without feed and water for 12 h (08:00-20:00) everyday.

\section{Caesarean section}

The dams were sacrificed under deep ether anesthesia on GD18. The numbers of total implantation sites on the wall of uterus as well as live and resorbed or dead fetuses were counted. Also, weights of placenta and male and female live fetuses were recorded.

After examination of live fetuses for the external anomalies, approximately one-half of the live fetuses 
were fixed in Bouin's solution for 1 week, and examined for the visceral abnormalities [24]. The remaining fetuses were fixed in $95 \%$ ethyl alcohol, cleared with $1 \%$ $\mathrm{KOH}$, stained with Alizarin red S, and examined for the skeletal malformations and variations [37].

\section{Data analysis}

Data obtained are expressed as the mean \pm SD or proportion (\%) of total number. Statistical analyses were performed by comparing the treatment groups with the vehicle control group using SAS software [33]. Continuous data variables such as maternal body weight, fetal body weight and placental weight were subjected to one-way analysis of variance (ANOVA), and Scheffe's multiple comparison test was conducted when analytic results were significant. The numbers of total implantations, and live and dead fetuses were statistically evaluated using the Kruskal-Wallis non-parametric ANOVA, followed by the Mann-Whitney U test when appropriate. Incidence data such as external, visceral and skeletal abnormalities were compared using the Fisher's exact probability test. Differences were considered statistically significant when $P<0.05$.

\section{Results}

\section{Maternal toxicity}

Restraint for $12 \mathrm{~h}$ caused a decrease in maternal body weights, leading to significant weight loss after 4 days of repeated stress (Fig. 1). The body weight loss recovered gradually after termination of the stress. However,

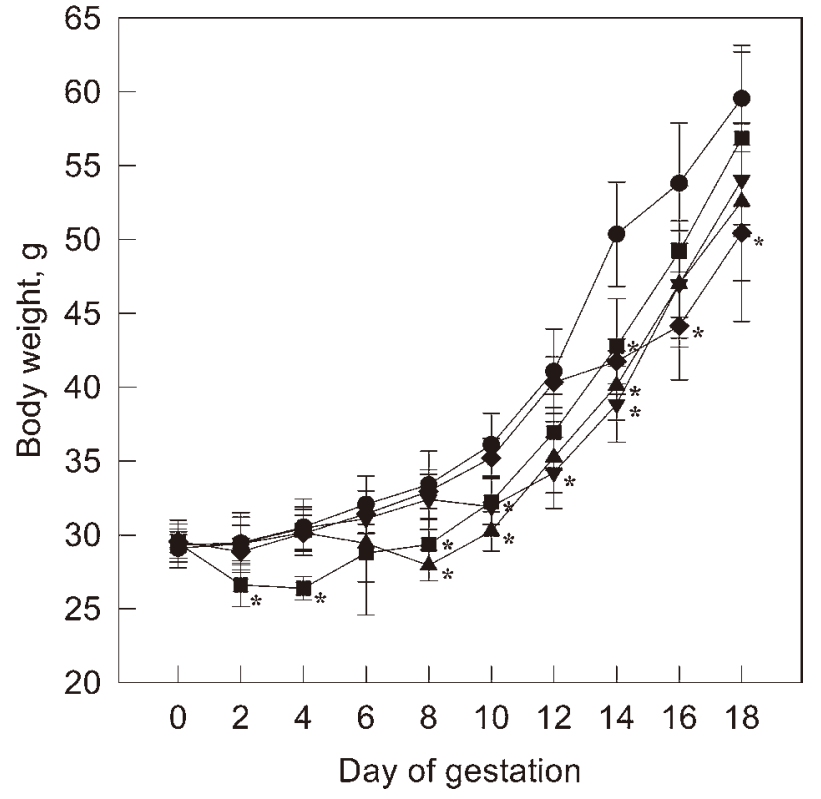

Fig. 1. Effect of restraint stress on maternal body weights. control (no stress); $\boldsymbol{\square}$, stress on days $1-4 ; \boldsymbol{\Lambda}$, stress on days $5-8 ; \boldsymbol{\nabla}$, stress on days $9-12 ; \bullet$, stress on days $13-16$. *Significantly different from control $(P<0.05)$.

most of the animals in the stressed groups had not fully recovered their body weights by the final day of gestation (GD18). Especially, the body weights of animals stressed on GD13-16 remained significantly low until the day of Caesarean section.

Restraint stress at all of the gestational stages did not affect embryonic implantation, which was confirmed by Caesarean section, and placental weights (Table 1). However, fetal death including resorption tended to in-

Table 1. Effect of restraint stress on reproductive findings

\begin{tabular}{|c|c|c|c|c|c|}
\hline \multirow{2}{*}{ Treatment } & \multirow{2}{*}{ Control } & \multicolumn{4}{|c|}{ Stress on gestational days } \\
\hline & & $1-4$ & $5-8$ & $9-12$ & $13-16$ \\
\hline Pregnant dams & 13 & 13 & 12 & 14 & 13 \\
\hline Implantation & $14.0 \pm 1.7$ & $15.2 \pm 2.4$ & $13.3 \pm 0.5$ & $13.9 \pm 0.9$ & $13.8 \pm 2.3$ \\
\hline Placental weight $(\mathrm{g})$ & $0.09 \pm 0.01$ & $0.10 \pm 0.01$ & $0.09 \pm 0.00$ & $0.09 \pm 0.01$ & $0.09 \pm 0.02$ \\
\hline Live fetuses & $13.7 \pm 1.7$ & $13.8 \pm 3.7$ & $12.5 \pm 0.6$ & $12.0 \pm 1.9$ & $11.0 \pm 2.3$ \\
\hline Early resorption & $0.15 \pm 0.38$ & $0.92 \pm 0.64 *$ & $0.50 \pm 0.67$ & $0.36 \pm 0.63$ & $0.46 \pm 0.66$ \\
\hline Late resorption & $0.15 \pm 0.38$ & $0.38 \pm 0.51$ & $0.33 \pm 0.49$ & $1.43 \pm 0.94 *$ & $2.23 \pm 1.36^{*}$ \\
\hline Death & 0 & $0.08 \pm 0.28$ & 0 & $0.14 \pm 0.36$ & $0.15 \pm 0.38$ \\
\hline Resorption and death (\%) & $2.2 \pm 4.5$ & $9.1 \pm 6.3$ & $6.3 \pm 6.3$ & $13.9 \pm 9.1^{*}$ & $20.6 \pm 11.0 *$ \\
\hline Body weights (g) Male & $1.47 \pm 0.14$ & $1.19 \pm 0.18^{*}$ & $1.34 \pm 0.07$ & $1.31 \pm 0.08^{*}$ & $1.22 \pm 0.13^{*}$ \\
\hline Female & $1.39 \pm 0.12$ & $1.14 \pm 0.18 *$ & $1.29 \pm 0.05$ & $1.25 \pm 0.13 *$ & $1.22 \pm 0.14 *$ \\
\hline
\end{tabular}

* Significantly different from control $(P<0.05)$. 
Table 2. Effect of restraint stress on fetal visceral abnormalities

\begin{tabular}{lccccc}
\hline \multirow{2}{*}{ Treatment } & \multirow{2}{*}{ Control } & \multicolumn{4}{c}{ Stress on gestational days } \\
\cline { 3 - 6 } & & $1-4$ & $5-8$ & $9-12$ & $13-16$ \\
\hline No. of litters examined & 13 & 13 & 12 & 14 & 13 \\
No. of litters affected $(\%)$ & $2(15.4)$ & $2(15.4)$ & $5(41.7) *$ & $7(50.0)^{*}$ & $3(23.1)$ \\
No. of fetuses examined & 92 & 91 & 77 & 84 & 76 \\
No. of fetuses malformed & $5(5 \%)$ & $2(2 \%)$ & $10(13 \%)^{*}$ & $15(18 \%)^{*}$ & $4(5 \%)$ \\
$\quad$ Ventricular dilatation & $0(0 \%)$ & $0(0 \%)$ & $5(6 \%)^{*}$ & $0(0 \%)$ & $0(0 \%)$ \\
$\quad$ Cleft palate & $0(0 \%)$ & $0(0 \%)$ & $0(0 \%)$ & $8(10 \%)^{*}$ & $0(0 \%)$ \\
Renal malposition & $5(5 \%)$ & $2(2 \%)$ & $6(8 \%)$ & $10(12 \%)$ & $4(5 \%)$ \\
\hline
\end{tabular}

*Significantly different from control $(P<0.05)$.

Table 3. Effect of restraint stress on fetal skeletal abnormalities

\begin{tabular}{lccccc}
\hline \multirow{2}{*}{ Treatment } & Control & \multicolumn{4}{c}{ Stress on gestational days } \\
\cline { 3 - 6 } & & $1-4$ & $5-8$ & $9-12$ & $13-16$ \\
\hline No. of litters examined & 13 & 13 & 12 & 14 & 13 \\
No. of litters affected (\%) & $4(30.8)$ & $8(61.5)^{*}$ & $9(75.0)^{*}$ & $8(57.1)^{*}$ & $7(53.8)^{*}$ \\
No. of fetuses examined & 86 & 88 & 73 & 84 & 66 \\
No. of fetuses malformed & $14(16 \%)$ & $32(36 \%)^{*}$ & $28(38 \%)^{*}$ & $26(31 \%)^{*}$ & $20(30 \%)^{*}$ \\
Extra ribs & $10(12 \%)$ & $7(8 \%)$ & $12(16 \%)$ & $12(14 \%)$ & $5(8 \%)$ \\
Vertebral abnormalities & $0(0 \%)$ & $0(0 \%)$ & $6(8 \%)^{*}$ & $0(0 \%)$ & $0(0 \%)$ \\
Sternal abnormalities & $8(9 \%)$ & $29(33 \%)^{*}$ & $15(21 \%)^{*}$ & $20(24 \%)^{*}$ & $17(26 \%)^{*}$ \\
\hline
\end{tabular}

*Significantly different from control $(P<0.05)$.

crease, showing significantly high early and late resorptions at the stages of GD1-4 and GD9-16, respectively. Moreover, the body weights of live fetuses in the stress groups were reduced, although there was no statistical significance at the stage of GD5-8.

\section{Developmental toxicity}

Only restraint stress on GD5-8 and GD9-12 induced cerebroventricular dilatation (6\%) and cleft palate (10\%), respectively (Table 2), leading to increased numbers of litters affected. In comparison, vertebral abnormalities (8\%), mainly bipartite (dumbbell-type) ossification, were observed only in animals stressed on GD5-8 (Table 3). Interestingly, stress at all of the gestational stages induced asymmetric or bipartite ossification of sternebrae, leading to a 2-fold increase in the number of malformed fetuses. The incidence of other anomalies or variations including renal malposition and costal malformations was not increased by restraint stress at any of the 4 stages.

\section{Discussion}

Restraint has been adopted as a standard stressor that imposes both physical and psychological demands on the subject [26]. This technique has been used in many areas of biology as a tool for understanding the basic physiological changes associated with stress.

In rats, there is some evidence for an association between restraint and implantation failure, but not for morphological abnormalities of fetuses [19], which may be due to reduced adrenocortical response to restraint in late pregnancy [16]. In comparison, it is known that restraint in the peri-implantation period leads to implantation failure, and that stress at appropriate times in organogenesis produces cleft palate, costal abnormalities and resorption of fetuses in mice [9, 14, 21, 28, 39]. Furthermore, some factors including type and duration of restraint, position and strain of mice, and lights are believed to be closely related to the incidence of maternal and fetal toxicities [15].

In the present study, we used a restraint stress model taping limbs of mice in a supine position for $12 \mathrm{~h}$ a day 
in a room with overhead lights on. This form of physical restraint was found to induce profound stress in ICR mice, resulting in body weight loss in dams and developmental anomalies in their fetuses [8, 10, 21, 28]. We attempted to elucidate the susceptibilities of embryos and fetuses at different developmental stages to the stress. Twelve-hour restraint caused severe body weight loss of dams over 4 days of repeated stress, influencing the final body weights in a manner which was similar to the body weight change induced by 12 -h restraint once on GD8 [21].

It is of interest to note that feed/water deprivation is stressful to rodents. It was reported that feed and water deprivation for up to $12 \mathrm{~h}$ induced a 2 -fold increase in blood corticosterone level, somewhat lower than the level ( $2.6-2.7$ folds) induced by the same duration of restraint stress [21]. In spite of the profound increase in blood glucocorticoids following feed and water deprivation, embryonic resorptions and fetal malformations were not observed [21], and there was a significant correlation between high maternal corticosteroid levels and the frequency of cleft palate in the offspring of restrained mice, but not in the feed-deprived animals [4]. Such results indicate that in addition to high levels of glucocorticoids, other factors such as energy depletion during physical struggling are involved in the development of fetal defects, as inferred from the prolonged loss of maternal body weights after 1- or 4-day restraint stresses, but not after feed and water deprivation [21, the present study]. Although control mice deprived of feed and water during daytime transiently lose body weight, it was reported that the animals took enough feed and water during nighttime, thereby recovering their body weights without fetal malformations [21].

Unexpectedly, restraint stress on GD1-4, the pre-implantation period, decreased fetal body weights and increased resorptions and skeletal abnormalities without influence on implantation. Although the mechanism behind this result remains to be clarified, it has been proposed that environmental imprinting factors such as hormonal imbalance might affect the fertilized ova, causing delayed embryonic resorptions, malformations and growth [39]. Restraint stress only on GD9-12 induced cleft palate of fetuses, indicative of the disturbing effect of glucocorticoids induced by the stress on the develop- ment of the palatine process. The closure of palatine processes in mice generally occurs on GD 13, and cleft palate is induced by chemicals including dexamethasone and dioxin when they are administered not later than GD13 [23]. It is of interest to compare the failure and occurrence of cleft palate in ICR mice subjected to 12-h restraint for 4 days on GD13 - 16 and in A/J mice exposed to 18-48-h stress once on GD14 [31, 32], respectively, which may be due to the intensity of stress and strain-difference. Although delayed ossification of vertebral bodies was observed after stress on GD5-8, similar to the observations of Miller and Chernoff [21], abnormalities of sternebrae were enhanced by stress at all of the gestational stages, suggestive of different time schedules in the formation and ossification of skeleton. Interestingly, the higher incidence of costal anomalies such as extra, fused or branched ribs observed by stress once on GD8 or GD9 $[9,21]$ was not reproduced by repeated restraint stress at any of the 4 stages.

Embryonic implantation was not affected by restraint stress at any of the gestational stages, in contrast to the implantation failure induced by 5 -h stress on GD1-6 in SW/B6D2F1 mice [39]. This shows a strain difference. It is noteworthy, however, that fetal mortality markedly increased after stress at the late stages of gestation. This was in agreement with enhanced post-implantation loss following stress for $14 \mathrm{~h}$ on GD9 in Swiss mice [11], or for 6-48 h on GD14 in A/J mice [31, 32]. Moreover, the body weights of live fetuses in the stress groups were decreased, although there was no statistical significance at the stage of GD5-8. Growth retardation, cleft palate and resorption of embryos and fetuses may have been caused by endocrine disturbances. It was demonstrated that stress caused activation of the adrenal axis and prolactin levels, whereas the reproductive hormonal axis including progesterone was inhibited [35, 38], and during pregnancy, maternal stress greatly enhanced blood glucocorticoids, especially at the late stage (2nd half) of gestation $[4,5,21]$. Also, the growth-retarding effect of glucocorticoids has been confirmed in primates including humans $[25,30,34]$.

As mentioned above, there are differences in the embryo-fetal toxicities of stress, according to animal species and strain, gestational period, and type, duration and intensity of stresses [15]. In addition to species, the 
duration of stress might be the key factor leading to fetal malformations. A longer duration of restraint produces a more severe effect. In susceptible mice, restraint stress longer than $12 \mathrm{~h}$ is generally required for the induction of growth retardation, malformation or resorption of fetuses. Relatively short duration ( $\leq 6 \mathrm{~h}$ ) did not induce fetal malformations at any gestational stage in both rats and mice $[2,12,13,38]$, but it did enhance the toxicity of other teratogens such as metals, retinoic acid, caffeine and aspirin [1, 2, 11, 12, 29].

The present study determined the susceptibility of dams and fetuses at different gestational stages to 4-day, 12-h restraint stress. Unexpectedly, the 4-day repeated stress did not induce implantation failure and costal malformations, but it did enhance the incidence of cleft palate, post-implantation loss and sternal abnormalities, in addition to growth retardation. In spite of the somewhat different aspects of fetal abnormalities observed by previous investigations [9, 14, 21, 28, 39], it should be emphasized that different outcomes in embryo-fetal toxicity were obtained by maternal stress at each gestational stage, pre- and peri-implantation, early and late organogenesis, and fetal development. A more detailed study using a highly susceptible mouse strain under a longer duration of stress might clarify the hypothetical hormonal mechanism involved in embryo-fetal toxicity.

\section{References}

1. Albina, M.L., Belles, M., Gomez, M., Sanchez, D.J., and Domingo, J.L. 2003. Influence of maternal stress on uraniuminduced developmental toxicity in rats. Exp. Biol. Med. 228: 1072-1077.

2. Albina, M.L., Colomina, M.T., Sanchez, D.J., Torrente, M., and Domingo, J.L. 2002. Interactions of caffeine and restraint stress during pregnancy in mice. Exp. Biol. Med. 227: 779-785.

3. Barlow, S.M., Knight, A.F., and Sullivan, F.M. 1978. Delay in postnatal growth and development of offspring produced by maternal restraint stress during pregnancy in the rat. Teratology 18: 211-218.

4. Barlow, S.M., McElhatton, P.R., and Sullivan, F.M. 1975. The relation between maternal restraint and food deprivation, plasma corticosterone, and induction of cleft palate in the offspring of mice. Teratology 12: 97-103.

5. Barlow, S.M., Morrison, P.J., and Sullivan, F.M. 1975. Effects of acute and chronic stress on plasma corticosterone levels in the pregnant and non-pregnant mouse. J. Endocrinol. 66: 90-99.
6. Barlow, S.M., Quyyumi, A.A., Rajaratnam, D.V., and Sullivan, F.M. 1976. Effects of stress and adrenocorticotrophin administration on plasma corticosterone levels at different stages of pregnancy in the mouse. Experimentia 32: 1480-1481.

7. Benedicktsson, R., Calder, A.A., Edwards, C.R., and Seckl, J.R. 1997. Placental 11 $\beta$-hydroxysteroid dehydrogenase: a key regulator of fetal glucocorticoid exposure. Clin. Endocrinol. 46: 161-166.

8. Beyer, P.E. and Chernoff, N. 1986. The induction of supernumerary ribs in rodents: role of the maternal stress. Teratogen. Carcinogen. Mutagen. 6: 419-429.

9. Chernoff, N., Kavlock, R.J., Beyer, P.E., and Miller, D.B. 1987. The potential relationship of maternal toxicity, general stress, and fetal outcome. Teratogen. Carcinogen. Mutagen. 7: 241-253.

10. Chernoff, N., Miller, D.B., Rosen, M.B., and Mattscheck, C.L. 1988. Developmental effects of maternal stress in the CD-1 mouse induced by restraint on single days during the period of major organogenesis. Toxicology 51: 57-65.

11. Colomina, M.T., Albina, M.L., Sanchez, D.J., and Domingo, J.L. 2001. Interactions in developmental toxicology: combined action of restraint stress, caffeine, and aspirin in pregnant mice. Teratology 63: 144-151.

12. Colomina, M.T., Esparza, J.L., Corbella, J., and Domingo, J.L. 1998. The effect of maternal restraint on developmental toxicity of aluminium in mice. Neurotoxicol. Teratol. 20 : 651-656.

13. Colomina, M.T., Roig, J.L., Torrente, M., Vicens, P., and Domingo, J.L. 2005. Concurrent exposure to aluminum and stress during pregnancy in rats: effects on postnatal development and behavior of the offspring. Neurotoxicol. Teratol. 27: 565-574.

14. deCatanzaro, D., MacNiven, E., Goodison, T., and Richardson, D. 1994. Estrogen antibodies reduce vulnerability to stress-induced failure of intrauterine implantation in inseminated mice. Physiol. Behav. 55: 35-38.

15. Golub, M.S., Campbell, M.A., Kaugman, F.L., Iyer, P., Li, L.-H., Donald, J.M., and Morgan, J.E. 2004. Effects of restraint stress in gestation: implications for rodent developmental toxicology studies. Birth Defects Res. Part B 71: 26-36.

16. Johnstone, H.A., Wigger, A., Douglas, A.J., Neumann, I.D., Landgraf, R., Seckl, J.R., and Russell, J.A. 2000. Attenuation of hypothalamic pituitary-adrenal axis stress responses in late pregnancy: changes in feedforward and feedback mechanisms. J. Neuroendocrinol. 12: 811-822.

17. Levine, S. and Treiman, D. 1964. Differential plasma corticosterone response to stress in four inbred strains of mice. Endocrinology 75: 142-144.

18. Loevy, H. 1963. Genetic influences on induced cleft palate in different strains of mice. Anat. Rec. 145: 117-122.

19. MacNiven, E., deCatanzaro, D., and Younglai, E.V. 1992. Chronic stress increases estrogen and other steroids in inseminated rats. Physiol. Behav. 52: 159-162.

20. Michel, C. and Fritz-Niggli, H. 1978. Induction of developmental anomalies in mice by maternal stress. Experimentia 34: 105-106. 
21. Miller, D.B. and Chernoff, N. 1995. Restraint-induced stress in pregnant mice-degree of immobilization affects maternal indices of stress and developmental outcome in offspring. Toxicology 98: 177-186.

22. Mosier, H.D., Jr., Dearden, L.C., Jansons, R.A., Roberts, R.C., and Biggs, C.S. 1982. Disproportionate growth of organs and body weight following glucocorticoid treatment of the fetus. Dev. Pharmacol. Ther. 4: 89-105.

23. Neubert, D. and Barrach, H.-J. 1977. Organotropic effects and dose-response relationship in teratology. pp. 405-412. In: Methods in Prenatal Toxicology (Neubert, D., Merker, H.-J., Kwasigroch, T.E. eds.), Georg Thieme Publishers, Berlin.

24. Nishimura, K.A. 1974. Microdissection method for detecting thoracic visceral malformations in mouse and rat fetuses. Congenit. Anom. 14: 23-40.

25. Novy, M.J. and Walsh, S.W. 1983. Dexamethasone and estradiol treatment in pregnant rhesus macaques: effects on gestation length, maternal plasma hormones and fetal growth. Am. J. Obstet. Gynecol. 145: 920-930.

26. Pare, W.P. and Glavin, G.B. 1986. Restraint stress in biomedical research: a review. Neurosci. Biobehav. Rev. 10: 339-370.

27. Prillaman, F. and Beck, S. 2003. Response of C57BL/6JBk, SWV/Bk, and their F1 outcrosses to immobilization stress. Birth Defects Res. Part B 68: 268.

28. Rasco, J.F. and Hood, R.D. 1994. Differential effect of restraint procedure on incidence of restraint-stress-induced rib fusion in CD-1 mice. Toxicol. Lett. 71: 177-182.

29. Rasco, J.F. and Hood, R.D. 1995. Enhancement of the teratogenicity of all-trans-retinoic acid by maternal restraint stress in mice as a function of treatment timing. Teratology 51: 63-70.

30. Reinisch, J.M., Simon, N.G., Karow, W.G., and Gandelman,
R. 1978. Prenatal exposure to prednisone in humans and animals retards intra-uterine growth. Science 202: 436-438.

31. Rosenzweig, S. and Blaustein, F.M. 1970. Cleft palate in $\mathrm{A} / \mathrm{J}$ mice resulting from restraint and deprivation of food and water. Teratology 3: 47-52.

32. Rosenzweig, S. 1966. Psychological stress in cleft palate etiology. J. Dent. Res. 45: 1585-1593.

33. SAS Institute, Inc. 1997. SAS/STAT Software: Changes and Enhancements through Release 6.12, SAS Institute, Cary.

34. Seckl, J.R. 1994. Glucocorticoids and small babies. Q. J. Med. 87: 259-262.

35. Seckl, J.R. 1997. Glucocorticoids, feto-placental 11 $\beta$-hydroxysteroid dehydrogenase type 2 , and the early life origins of adult disease. Steroids 62: 89-94.

36. Servatius, R.J., Natelson, B.H., Moldow, R., Pogach, L., Brennan, F.X., and Ottenweller, J.E. 2000. Persistent neuroendocrine changes in multiple hormonal axes after a single or repeated stressor exposures. Stress 3: 263-274.

37. Staples, R.E. and Schnell, V.L. 1968. Refinements in rapid clearing technique in the $\mathrm{KOH}$-alizarin red $\mathrm{S}$ method for fetal bone. Stain Technol. 43: 61-63.

38. Tyl, R.W., Ballantyne, B., Fisher, L.C., Fait, D.L., Savine, T.A., Pritts, I.M., and Dodd, D.E. 1994. Evaluation of exposure to water aerosol or air by nose-only or whole-body inhalation procedures for CD-1 mice in developmental toxicity studies. Fund. Appl. Toxicol. 23: 251-260.

39. Wiebold, J.L., Stanfield, P.H., Becker, W.C., and Hillers, J.K. 1986. The effect of restraint stress in early pregnancy in mice. J. Reprod. Fertil. 78: 185-192.

40. Zimmerman, E.F. 1997. Palate. p. 183. In: Drug Toxicity in Embryonic Development (Kavlock, R.J. ed.), Springer, New York. 